\title{
Personalização de um sistema operacional linux para projeto de doação de notebooks para alunos em situação de ensino remoto emergencial
}

Pablo João Canal da Costa*, Adriano Canabarro Teixeira**

\section{Resumo}

Este artigo relata o processo de personalização de um sistema operacional baseado em Linux voltado a finalidades educacionais que atendam a demanda de um projeto local de captação de notebooks, na rede municipal de ensino de Passo Fundo - RS. O trabalho possui caráter descritivo, tendo por objetivo relatar o desenvolvimento do projeto. O sistema operacional recebeu o nome de Gepid OS (GEPID OS, 2021), em referência ao Grupo de Pesquisa em Cultura Digital da Universidade de Passo Fundo - RS, contendo pré-instalado uma série de pacotes de programas com fins educacionais. Este sistema, baseado no Linux Xubuntu LTS, passou a ser instalado de forma experimental em notebooks do projeto Banco de Notebooks, voltado à arrecadação e doação de notebooks para alunos da rede pública municipal de ensino em situação de ensino remoto emergencial, que não dispõe de dispositivos adequados para a realização das aulas on-line. Em uma segunda etapa de desenvolvimento, o sistema experimental Gepid OS passou a fazer parte de um projeto coordenado pelo programa EducaTech PF da Secretaria Municipal de Educação de Passo Fundo, contribuindo para o desenvolvimento de um sistema operacional Linux mais completo, voltado a notebooks adquiridos para a rede municipal de ensino.

Palavras-chave: educação; tecnologias educacionais. inclusão digital. software livre; Linux.

* Mestre em Educação pelo PPGEdu UPF. Especialista em Linguagens e Tecnologias na Educação (IFSul). Graduado em Psicologia (UPF). E-mail: pablojoaoc@gmail.com

* Professor do Programa de Pós Graduação em Educação (PPGEdu-UPF). Doutor em Informática na Educação (UFRGS). E-mail: teixeira@upf.br

https://10.5335/rbecm.v5iespecial.13270

http://creativecommons.org/licenses/by-nc-nd/4.0

ISSN: 2595-7376 


\section{Introdução}

Este artigo traz um relato de experiência sobre a personalização de um sistema operacional Linux, voltado para atender as necessidades educacionais de alunos em situação de ensino remoto emergencial na rede pública de educação da cidade de Passo Fundo - RS, atendidos por um projeto de captação de notebooks destinado a fins escolares, chamado Banco de Notebooks.

Durante 2020, o primeiro ano afetado pela pandemia decorrente do coronavírus Covid-19, o mundo experienciou momentos onde o estilo de vida precisou ser bruscamente alterado. Fábricas, comércios e escolas precisaram parar totalmente em vários lugares no mundo, inclusive no Brasil, na tentativa de frear a disseminação do vírus. Alguns desses serviços voltaram a funcionar com medidas mais restritivas, porém as escolas permaneceram fechadas, sem aulas presenciais. Ao longo de 2020 o modelo de ensino remoto emergencial foi adotado por grande parte das instituições que antes eram predominantemente voltadas ao ensino presencial. Nas escolas de Ensino Fundamental públicas que, em geral, atendem alunos sendo parte oriundos de famílias de baixa renda, a migração para o ensino remoto mostrou-se mais complicado e em muitos casos alunos ficaram sem nenhum tipo de aula ou mesmo que conseguissem realizar seus estudos. Entre as dificuldades, podemos citar o acesso precário, ou ausente, a equipamentos tecnológicos necessários para acompanhar aulas remotas, como computadores, notebooks, tablets ou smartphones.

Tais dificuldades foram também sentidas na rede pública de ensino de Passo Fundo, onde se situa este trabalho. Diante desta demanda por computadores, em especial notebooks para estudo remoto, surgiu um projeto visando captar notebooks usados, prepará-los e destiná-los para alunos da rede pública que estivessem em situação de ensino remoto, e que não tinham acesso a computador para realizar os estudos e/ou acompanhar aulas. Nisto, surgiu a necessidade do desenvolvimento de um sistema operacional baseado em software livre e voltado a fins educacionais/escolares, e, ainda, que consiga reunir elementos úteis e adaptados para a rede de ensino local.

\section{Linux}

Linux ou GNU/Linux, é o nome que se dá a sistemas operacionais de computador construídos sob o Kernel Linux, um núcleo operacional desenvolvido pelo 
programador finlandês Linus Torvalds (1997). Diferente de sistemas operacionais proprietários de código fechado, como o Windows ou Mac OS, o Linux se fundamenta na filosofia do software livre, proposta por Richard Stallman (2010). Software livre não é sinônimo de gratuito, apesar de que os sistemas operacionais Linux e seus softwares sejam disponibilizados gratuitamente, porém o conceito vai muito além.

Segundo a filosofia da Free Software Foundation (2021), para um programa qualquer ser considerado software livre, este deve conceder liberdade para o usuário poder acessar o código-fonte, ou seja, as linhas de programação, poder estudá-lo e até mesmo modificá-lo, se assim o quiser. Além disso, o usuário deve ter a liberdade de redistribuir cópias deste programa, com ou sem alterações.

São estas liberdades que permitem que um software livre possa ser construído coletivamente, onde comunidades de programadores se unem para desenvolver um software ou mesmo lançar uma versão melhorada de um software livre já existente. É por isso que qualquer usuário ou grupo pode criar suas próprias variantes de um sistema operacional baseado em Linux. Isto traz por um lado, uma ampla diversidade de remasterizações de distribuições Linux, com caras e funcionalidades diferentes, e por outro, torna mais complexa a escolha de qual Linux o usuário optará. Algumas distribuições mais consagradas, que garantem melhor suporte, tendem a se tornarem mais relevantes, porém nem sempre conseguem agradar a todos os usuários, ou oferecer o que cada um em sua particularidade necessite. E o mundo Linux traz a possibilidade de se reconfigurar essas distribuições, e relançá-las com outros nomes e características.

\section{Personalização do sistema operacional}

Neste projeto foi desenvolvida uma versão personalizada de um sistema Linux, baseado no UBUNTU (2021), versão estável de número 20.04. Como diferenciais do sistema base, o Linux voltado para o projeto contou com uma identidade visual própria, personalizada a partir da interface gráfica XFCE (2021), configurada para ser simples e intuitiva. Com isso, manteve-se a leveza necessária para funcionar em notebooks mais antigos e modestos, como os que possuem processador de cerca de $1.5 \mathrm{Ghz}$ e memória RAM de 2 GB. A partir da personalização do sistema, foi gerada uma imagem do tipo ISO, com instalador próprio. O sistema foi nomeado 
de GEPID OS (2021) em referência ao Grupo de Pesquisa em Cultura Digital da Universidade de Passo Fundo, de onde surgiu a ideia do projeto.

Para esta imagem de sistema, foram selecionados programas e jogos relacionados à área educacional, que já vem instalados e prontos para uso. Entre eles a plataforma de desenvolvimento e programação Scratch; as plataformas de jogos educacionais Gcompris, Tuxmath e Gbrainy; a tabela periódica Kalzium; o programa de astronomia KStars; o pacote de escritório LibreOffice; e mais jogos voltados ao desenvolvimento do raciocínio lógico e/ou criatividade, como o cubo mágico Pybik, o jogo explorador de ambientes virtuais Minetest, o programa de projetos em peça tipo lego Leocad; entre outros recursos. Como diferencial sobre outras distribuições Linux para fins educacionais, o Gepid OS traz documentos sobre segurança digital para crianças e adolescentes, com tutoriais de bons usos da internet, acessíveis desde o desktop do sistema.

\section{Figura 1: Interface do Gepid OS}

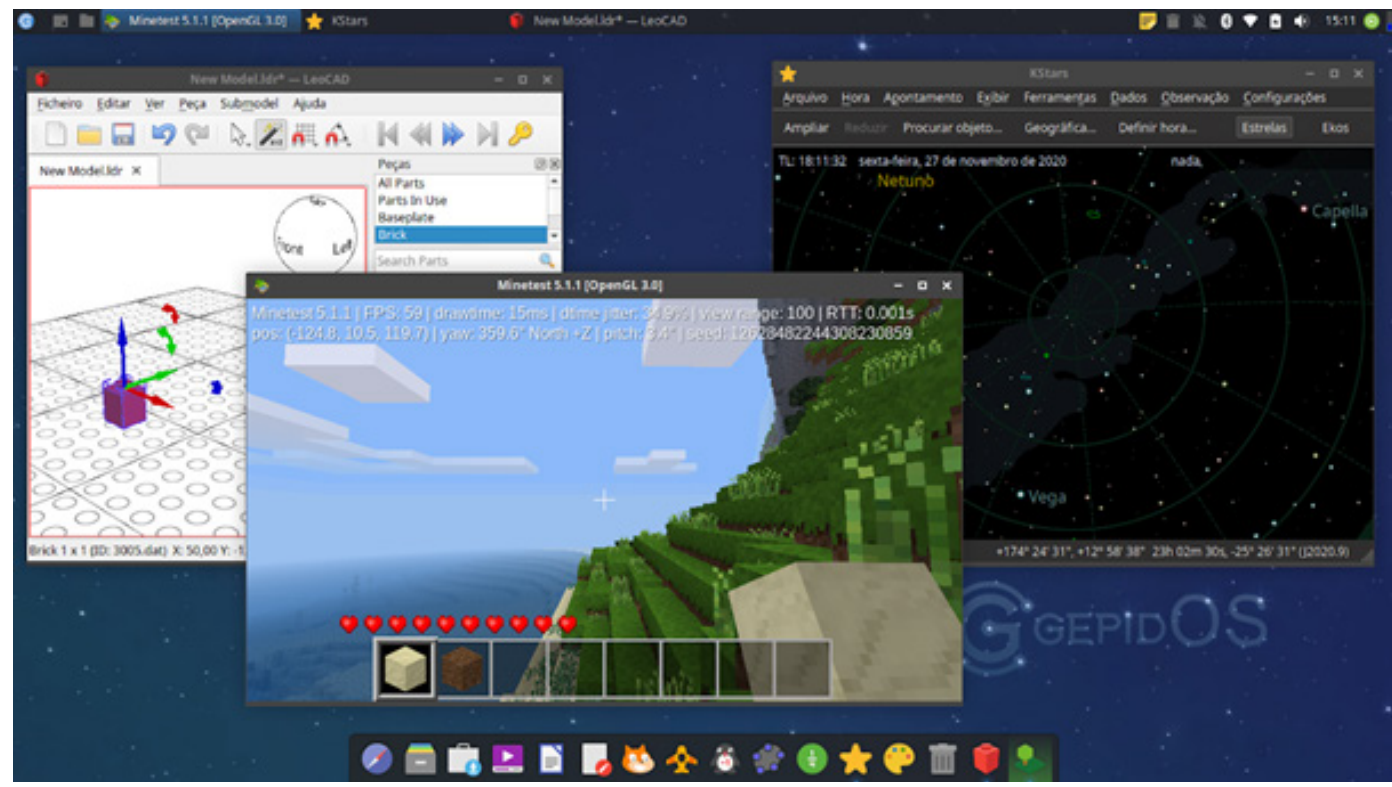

Fonte: GEPID OS, 2021

O Gepid OS passou a ser instalado de forma experimental em notebooks do projeto Banco de Notebooks. Foi criado um site de apresentação do sistema e do projeto de arrecadação de notebooks, ligado ao Grupo de Pesquisa em Cultura Digital da 
Universidade de Passo Fundo, com apoio do Prisma Espaço Geek, definido como o ponto de coleta dos aparelhos, formatação e instalação do sistema.

Com o decorrer deste trabalho, o sistema operacional Gepid OS passou a ser integrado ao programa EducaTech PF, ligado à Secretaria Municipal de Educação de Passo Fundo. Para este projeto, somaram-se também contribuições de outro sistema operacional baseado em Linux elaborado durante pós-graduação em Linguagens e Tecnologias na Educação no Instituto Federal Sul Rio-Grandense de Passo Fundo, chamado Sabiá OS. Este último conta em especial com recursos e programas voltados a professores criadores de conteúdo digital, como os editores de imagens GIMP e Inkscape, platafoma de streaming e gravação de videoaulas OBS Studio, além de uma loja de aplicativos online gratuitos, voltadas a professores criadores de conteúdo digital.

Aspectos diferenciais desses dois sistemas, Gepid OS e Saibá OS, compõe os recursos disponíveis no sistema operacional Linux proveniente do programa $E d u$ caTech $P F$. Este encontra-se agora em fase de testes, recebendo o nome provisório de EducaTech OS.

\section{Considerações finais}

Atualmente o projeto Banco de Notebooks passou a fazer parte de um projeto maior, chamado Criança Conectada (EDUCA PF, 2021), que conta com parceria do Ministério Público do Estado do Rio Grande do Sul e da Fundação Universidade de Passo Fundo. Este projeto busca captar, além de computadores e notebooks, celulares irregulares apreendidos pelo poder público, que passam a ser regularizados e reaproveitados para fins educacionais através de doação para alunos da rede municipal de ensino. Através do programa EducaTech, o sistema operacional que está sendo organizado está destinado a ser instalado em cerca de 2000 notebooks novos adquiridos para escolas da rede municipal de ensino de Passo Fundo. 


\section{Customizing a linux operating system for a notebook donation project for students in emergency remote teaching situation}

\section{Abstract}

This article reports the customization process of a Linux-based operating system aimed at educational purposes that meet the demand of a local project for capturing notebooks, in the municipal education network in Passo Fundo - RS. The work has a descriptive character, aiming to report the development of the project. The operating system was named Gepid OS, in reference to the Digital Culture Research Group (GEPID) of the University of Passo Fundo - RS, containing pre-installed a series of program packages with educational purposes. This system, based on Linux Xubuntu LTS, started to be installed on an experimental basis in notebooks of the Bank of Notebooks project, aimed at collecting and donating notebooks for students in the municipal public education network in emergency remote education situations, which do not have a devices suitable for conducting online classes. In a second stage of development, the Gepid OS experimental system became part of a project coordinated by the EducaTech PF program of the Municipal Education Department of Passo Fundo, contributing to the development of a more complete Linux operating system, aimed at notebooks purchased for the municipal education system.

Keywords: Education; Educational technologies. Digital inclusion. Free software; Linux.

\section{Referências}

GEPID OS. Disponível em: <http://gepid.upf.br/os>. Acesso em 30 de novembro de 2021.

EDUCA PF. Criança Conectada, 2021. Disponível em: <https://www.educapf.org/criancaconectada>. Acesso em 09 de dezembro de 2021.

FREE SOFTWARE FOUNDATION. Filosofia do Projeto GNU. Free Software Foundation, 2021. Disponível em: <https://www.gnu.org/philosophy/philosophy.pt-br.html> Acesso em 05 de março de 2021.

STALLMAN, Richard. Free Software Free Society: Selected Essays of Richard Stallman. Boston, MA. Free Software Foundation, 2 ed, 2010.

TORVALDS, Linus. Linux: a portable operating system. Helsinki, University of Helsinki, 1997.

UBUNTU. Disponível em: <https://ubuntu.com/> Acesso em 30 de novembro de 2021.

XFCE. Disponível em: <https://www.xfce.org/> Acesso em 30 de novembro de 2021. 